\title{
Desorption Electrospray Ionization Mass Spectrometry for Monitoring the Kinetics of Baeyer-Villiger Solid-State Organic Reactions
}

\author{
Ying Xie, Li-Fang He, Shui-Chao Lin, Hai-Feng Su, Su-Yuan Xie, \\ Rong-Bin Huang, and Lan-Sun Zheng \\ State Key Laboratory for Physical Chemistry of Solid Surfaces, and Department of Chemistry, College of \\ Chemistry and Chemical Engineering, Xiamen University, Xiamen, China
}

\begin{abstract}
Desorption electrospray ionization mass spectrometry (DESI-MS) has been used for monitoring solid-state organic reaction in ambient air, specifically the Baeyer-Villiger (BV) type reaction involving the oxidation of ketones (benzophenone or deoxybenzoin) by m-chloroperbenzoic acid (m-CPBA) in solid-state. The DESI mass spectra obtained at regular intervals during the BV reaction processes are featured, with the amount of ester products increasing as those of ketone reactants decrease. Quantitative analyses of relative intensities of the product, made to quantify the reaction degree of typical solid-state organic reaction (SSOR), show a precision with RSDs of around 5\% to $12 \%$, though the RSDs for direct analysis of intensities of the reactant or the product in the solid-state are obviously larger. The kinetics of the BaeyerVilliger type reactions in solid-state are shown to be dramatically different, in reaction rate, kinetic curve, as well as concentration dependence, from those of the same reactions taking place in solution. (J Am Soc Mass Spectrom 2009, 20, 2087-2092) (c) 2009 American Society for Mass Spectrometry
\end{abstract}

$\mathrm{T}$ The recent breakthrough discovery of desorption electrospray ionization (DESI) [1] allows direct and rapid mass spectrometry (MS) analysis of various compounds in solid surfaces, free from redundant sampling preparation [2-8]. It has been adopted for mapping analytes separated by thin-layer chromatography [9], profiling (linear and 2D imaging) biological tissue analysis semiquantitatively in situ [10], imaging latent fingerprints chemically [11], determining SVOC in aerosols rapidly [12], and analyzing liquid samples directly [13]. Shortly after the discovery of DESI, a number of ambient ionization MS methods were reported for direct analysis of solid samples, including direct analysis in real time (DART) [14], desorption atmospheric pressure chemical ionization (DAPCI) [15], matrix-assisted laser desorption electrospray ionization (MALDESI) [16], desorption atmospheric pressure photoionization (DAPPI) [17], electrospray-assisted laser desorption/ionization (ELDI) [18], and atmosphericpressure solids analysis probe (ASAP) [19]. All of these MS techniques, as well as DESI-MS, are potentially applicable for direct following solid-state organic reactions (SSORs) [20, 21]. To our knowledge, however, effort focusing on MS monitoring of SSOR has never been discussed heretofore. Here we reported the first application using DESI-MS for direct characterization of

Address reprint requests to Dr. S.-Y. Xie, State Key Laboratory for Physical Chemistry of Solid Surfaces, and Department of Chemistry, College of Chemistry and Chemical Engineering, Xiamen University, Xiamen 361005, China. E-mail: syxie@xmu.edu.cn kinetics of two representative SSORs, i.e., the BaeyerVilliger (BV) $[22,23]$ type reactions of $m$-chloroperbenzoic acid (m-CPBA) with benzophenone (PhCOPh, 1) or deoxybenzoin $\left(\mathrm{PhCOCH}_{2} \mathrm{Ph}, 2\right)$. Unexpected kinetics of the $\mathrm{BV}$ reactions in solid-state, including reaction rate, kinetic curve, and concentration dependence, have been revealed to be different from those of the same reactions in solution.

\section{Experimental}

\section{Materials and Chemicals}

Benzophenone (98\%) and chloroform (99\%) were purchased from Sinopharm Chemical Reagent Co. (Shanghai, China). Deoxybenzoin (97\%) was obtained from Alfa Aesar, a Johnson Matthey Co. (Ward Hill, MA, USA). m-CPBA (85\%) was purchased from Refine Chemicals Science and Technology Developing Co. (Tianjin China). HPLC-grade methanol was obtained from TEDIA Co. (Fairfield, OH, USA).

\section{Monitoring the BV Reaction by DESI-MS}

The experiments were conducted at the temperature around $22{ }^{\circ} \mathrm{C}$. About $0.2 \mathrm{~g}$ of ketone ( $\mathbf{1}$ or 2$)$ was mixed together with 2 mol equiv of m-CPBA to afford the reactant mixture. For the concentration-dependent experiments, the reactants "concentration" (60 and 80 $\mathrm{wt} \%)$ of $\mathbf{2}$ and $\mathrm{m}-\mathrm{CPBA}$ were changed depending on the 
loading amounts of amylum powder (serving as diluent) added into the reactants mixture ( 2 and $\mathrm{m}$ CPBA). The mixture of reactants was ground in an agate mortar for $10 \mathrm{~min}$, followed by being pressed in pellets using a Qwik Handi-Press (Thermo Electron Corp., Waltham, Greater London, UK) for kinetic studies using DESI-MS. The pellets of reactants were kept in air at room-temperature (around $22^{\circ} \mathrm{C}$ ) unless analyzed by the DESI-MS in regular intervals of time (intervals of $2 \mathrm{~h}$ for 1 and $4 \mathrm{~h}$ for 2 ). The DESI-MS analyses, recorded in the positive ion mode by a Bruker Esquire HCT ion trap mass spectrometer (Billerica, MA, USA) coupled with a home-made DESI device, were run just a short time (less than $5 \mathrm{~s}$ ) every $2 \mathrm{~h}$ for oxidation of $\mathbf{1}$ (or every $4 \mathrm{~h}$ for 2 ). In the experiment, sample surfaces previously exposed to the DESI analyses were not used as the spots for the next DESI determination.

Parameters of the DESI source were optimized to enhance the signal intensity. Methanol was used as the spray solvent at a flow rate of $0.16 \mathrm{~mL} / \mathrm{h}$. The angle between the DESI spray needle and the sample surface was $86^{\circ}$; the tip of the spray needle was $5 \mathrm{~mm}$ from the surface of the sample; the end of the MS-inlet capillary was about $6 \mathrm{~cm}$ from the sample, the pressure of nebulizing nitrogen gas was 60 psi, the capillary voltage was $1 \mathrm{kV}$, the flow rate of desolvation gas was $1 \mathrm{~L} / \mathrm{min}$, and the temperature of desolvation gas was $200{ }^{\circ} \mathrm{C}$ around the entry of MS-inlet capillary.

The experiments of the BV reaction in solution were carried out starting from ketones [1 $\mathrm{g}$ of $\mathbf{1}, 1 \mathrm{~g}$ of 2 (for $0.02 \mathrm{~g} / \mathrm{mL}$ ), or $0.5 \mathrm{~g}$ of 2 (for $0.01 \mathrm{~g} / \mathrm{mL}$ )] and $2 \mathrm{~mol}$ equiv of m-CPBA in $50 \mathrm{ml}$ of $\mathrm{CHCl}_{3}$. The solution was analyzed by electrospray ionization (ESI)-MS during the reaction.

\section{Results and Discussion}

\section{Baeyer-Villiger Reactions Detected by DESI-MS}

Chemists carry out organic synthesis in solution traditionally, but a number of organic reactions have been reported to take place well in solventless solid-state [24, 25]. Commercial applications based on SSOR have been stimulated because of their remarkable advantages, e.g., reduced pollution, low cost, and simplicity in handling [26]. One typical example of SSOR is the BV type reaction involving oxidation of ketones (e.g., PhCOR, $\mathrm{R}=$ $\mathrm{CH}_{2} \mathrm{Ph}, \mathrm{Ph}, \mathrm{p}$-tolyl) by $\mathrm{m}$-chloroperbenzoic acid (mCPBA) [17, 18]. Challenges are to explore the kinetic properties of these SSORs, which are different from those of their counterpart reactions in solution and to provide illumination for investigation of other kinetic reactions on solid surfaces such as heterogeneous catalysis and electrode reactions. Partly due to the difficulties in monitoring the solid reactants and products in real time, however, detailed kinetic information of the BV reaction in the solidstate, as well as most of the other SSORs, is unclear yet.

X-ray diffraction [27], nuclear magnetic resonance [28], and photometric methods $[29,30]$ were reported for following the kinetic processes of some SSORs, but these methods work only if the products exhibit characteristic signals quite different from those of the reactants. Moreover, quantifiable detection in situ is prevented by the laborious sampling processes for these traditional analyses. Therefore, only a little kinetics information of SSORs was obtained from the traditional measurements. However, sampling-free mass spectrometry, such as DESI-MS, can be a versatile tool for following ambient SSORs because of its capability for clear characterizations and quantification of molecular composition at surfaces in ambient air.

Figure 1a shows a series of representative DESI-MS spectra of the BV SSOR for $\mathbf{1}$ acquired at different reaction stages in real time. Characteristic ions of the ketone reactant 1 and the ester product $\mathrm{PhCO}_{2} \mathrm{Ph} 3$ are detected in the forms of their sodium or hydrogen adducts in the positive ion mode. Shown in Figure $1 \mathrm{~b}$ is a graph depicting the relative intensities of $\mathbf{1}$ or $\mathbf{3}$ as a function of reaction time, from which the kinetic feature is clearly shown with the amounts of product 3 increasing as those of reactants 1 decrease during the BV reactions. Similar kinetic process of the $\mathrm{BV}$ reaction from reactant 2 to product $\mathrm{PhCO}_{2} \mathrm{CH}_{2} \mathrm{Ph} 4$ in the solid-state is also recorded (Supplementary data-1, which can be found in the electronic version of this article).

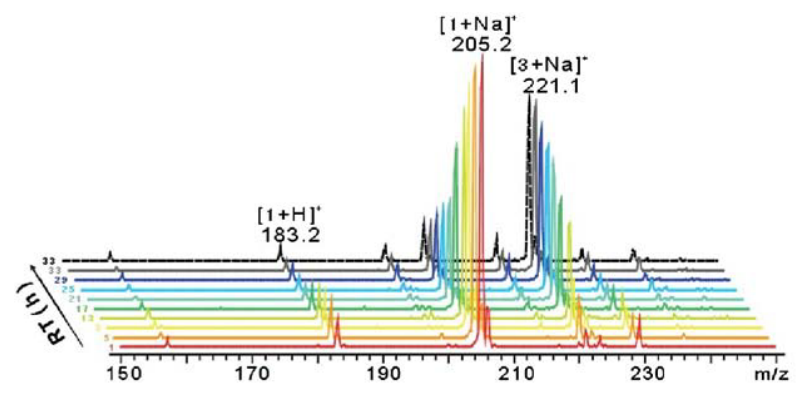

(a)

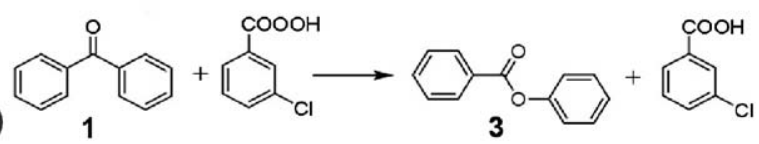

(b)

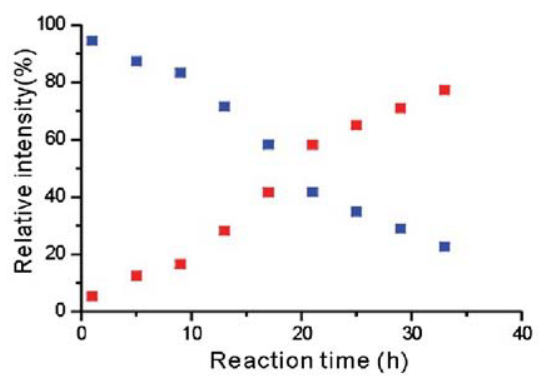

Figure 1. (a) MS spectra acquired during the BV SSORs involving $\mathrm{m}$-chloroperbenzoic acid with benzophenone. The intensities of the mass peaks are normalized to the same sum of ketone reactant and ester product. The aftermost MS spectra plotted in black dash lines are those of no-DESI-monitoring samples. RT: Reaction Time. (b) The relative intensities of $\mathbf{1}$ (blue rectangles) or 3 (red rectangles) as a function of reaction time. 
The kinetic processes of the BV SSORs are concerned to be influenced by the repeated DESI-monitoring because: (1) the DESI might spray methanol solution on the surface of the reactant pellet to allow a conventional solution phase (thin film) reaction to proceed; (2) the desolvation gas $\left(200{ }^{\circ} \mathrm{C}\right)$ of the DESI might impose additional heat onto the pellet surface so as to accelerate the SSORs. In those cases, the BV reactions in the interior of the pellets should be different from the reactions on the surface exposed to the DESI. However, the equivalent spectra acquired from both surface and interior of the pellets of BV reaction have shown the identical results, and the kinetics of BV SSORs seems to be free from the influence of short-time DESI-MS monitoring (Supplementary data-2). This conclusion is corroborated by the similar MS spectra of regular DESI-monitoring sample and no-DESI-monitoring sample as shown in Figure 1 and Supplementary data-1. The DESI tolerance of the SSORs is possibly attributable to the fact that the time for DESI-monitoring (a few seconds) is too fast to influence the long-standing BV reactions (10 h).

\section{RSDs of DESI-MS Quantitative Analysis for Baeyer-Villiger Reactants/Products}

To quantify the degree of the BV reaction, the amounts of $\mathrm{BV}$ reactants/products changing in different reaction periods are analyzed by DESI-MS directly. Possibly owing to a variable amount of analyte into the mass spectrometer in the DESI-MS process, however, the RSDs are large for direct quantitative analysis of ketone reactants or ester products based on the DESI-MS intensity signals. As listed in Table 1 , the RSDs could be more than $40 \%$ as calculated by averaging with all the DESI-MS data acquired during the BV oxidation reaction in solid-state. The DESI-MS analysis of each sample was repeated for at least three times (Supplementary data-3).

To detour the large RSDs for direct monitoring of BV SSOR reactants/products, the concept of "internal standard" [31] are utilized. Due to the similarity of ionization efficiencies of ketone reactants and ester products, as shown in their mass spectra (Figure 1), the relative intensity of the ester product in the mixture of solid sample (ester product plus ketone reactant) can be quantified having higher precision and reproducibility with RSDs less than $12 \%$ (Table 1). Taking the SSOR of $\mathbf{1}$ as an example (Supplementary data-3); the average RSD is as low as $7.1 \%$ for determining the relative intensity of the product, made to quantify the reaction degree of the typical solid-state organic reaction.

\section{Oxidation of Ketones (Deoxybenzoin and Benzophenone) During the DESI Process}

Shown in Figure 2a is the classic electrospray mass spectrum (ESI-MS) of $\mathbf{2}$ in methanol solution, in which no oxidized species of $\mathbf{2}$ is detected. By contrast, however, the characteristic mass-to-charge signal of $4 \mathrm{(m} / \mathrm{z}$
Table 1. RSDs of the DESI-MS analyses for the BV oxidation reactions

\begin{tabular}{|c|c|c|c|}
\hline \multirow[b]{2}{*}{$\begin{array}{l}\text { The composition of the } \\
\text { starting reactants for } \\
\text { BV reaction }\end{array}$} & \multicolumn{3}{|c|}{$\mathrm{RSDs}^{\mathrm{a}}$} \\
\hline & $\begin{array}{l}\text { Reactant }^{\mathrm{b}} \\
(\%)\end{array}$ & $\begin{array}{l}\text { Products } \\
(\%)\end{array}$ & $\begin{array}{c}\text { Relative } \\
\text { intensities of } \\
\text { products (\%) }\end{array}$ \\
\hline $\begin{array}{l}35 w t \% \text { of } 1,65 w t \% \text { of } \\
\text { m-CPBA }\end{array}$ & 41.8 & 45.4 & 7.1 \\
\hline $\begin{array}{l}36 w t \% \text { of } 2,64 w t \% \text { of } \\
\text { m-CPBA }\end{array}$ & 54.4 & 58.6 & 11.9 \\
\hline $\begin{array}{l}29 \text { wt } \% \text { of } 2,51 \text { wt } \% \\
\text { m-CPBA, } 20 \text { wt } \% \text { of } \\
\text { diluent }^{d}\end{array}$ & 47.3 & 45.9 & 9.6 \\
\hline $\begin{array}{l}22 \text { wt } \% \text { of } 2,38 w t \% \\
\text { m-CPBA, } 40 \% \text { of } \\
\text { diluent }\end{array}$ & 43.9 & 47.4 & 5.4 \\
\hline
\end{tabular}

aThe RSDs (relative standard deviations) are the average value of the RSDs calculated at different concentration levels of reactants (1 or 3 ) and products ( 2 or $\mathbf{4}$ ) during the BV reactions. Taking the BV reaction involving 1 with $\mathrm{m}$-CPBA as an example, a total of 18 sets of MS intensity data were recorded in every $2 \mathrm{~h}$ during the $33 \mathrm{~h}$ of the BV reaction. For each set of data, the mass spectra were repeatedly detected for three times within a minute, and the RSDs can be calculated for reactant 1 , product 3 , and relative intensity $[3 /(1+3)]$. Consequently, 18 RSDs are acquired. The RSDs listed for the reaction involving 1 with $\mathrm{m}$-CPBA in the table are the average values of the 18 RSDs. The details of the intensities and RSDs for the model BV reaction have been listed in Table S1 in Supplementary data-3.

b1 $(m / z 205)$ or $2(m / z 219)$.

c3 $(m / z 221)$ or $4(m / z 235)$.

${ }^{\mathrm{d}}$ Amylum powder is used as diluent loaded into the reactant mixture (2 and $\mathrm{m}$-CPBA) for adjusting the "concentration."

235, assigned to sodium adduct of oxidized product from 2) appears in the DESI-MS of a newly-made solid pellet of pure 2 (Figure $2 b$ ). That is, even in the absence of oxidant $\mathrm{m}-\mathrm{CPBA}$, ketone reactant 2 can be oxidized to form ester product 4 in the DESI-MS experiment. There are two possible reasons for this evidence: (1) 2 is oxidized in air during its pellet-sampling process; (2) oxidation of $\mathbf{2}$ is taking place simply in the DESI process. The former possibility of oxidation occurrence in the sampling process, however, can be ruled out by the following experiments: the above-mentioned newlymade sample of the solid pellet of 2 , which had undergone the DESI detection process and shown a clear MS peak of $m / z 235$, was dissolved in methanol and analyzed by electrospray ionization-MS (ESI-MS). The $\mathrm{m} / \mathrm{z}$ 235 disappears completely in the acquired electrospray ionization mass spectrum (Figure 2c), which looks the same as Figure 2a recorded for no-DESI-monitoring sample of $\mathbf{2}$ in solution. It is thus established that the oxidation is a result of solely the DESI process. This conclusion has been confirmed by the DESI-MS experiment with a pellet of just the reactant 2 and monitoring the oxidation product 4 as a function of time. The abundance of both the product and reactant are equivalent over a long period of time (Supplementary data-4).

As for benzophenone (1), the oxidation during the DESI operation was also observed, but its degree of oxidation was much less than that of 2 (Supplementary data-5). 


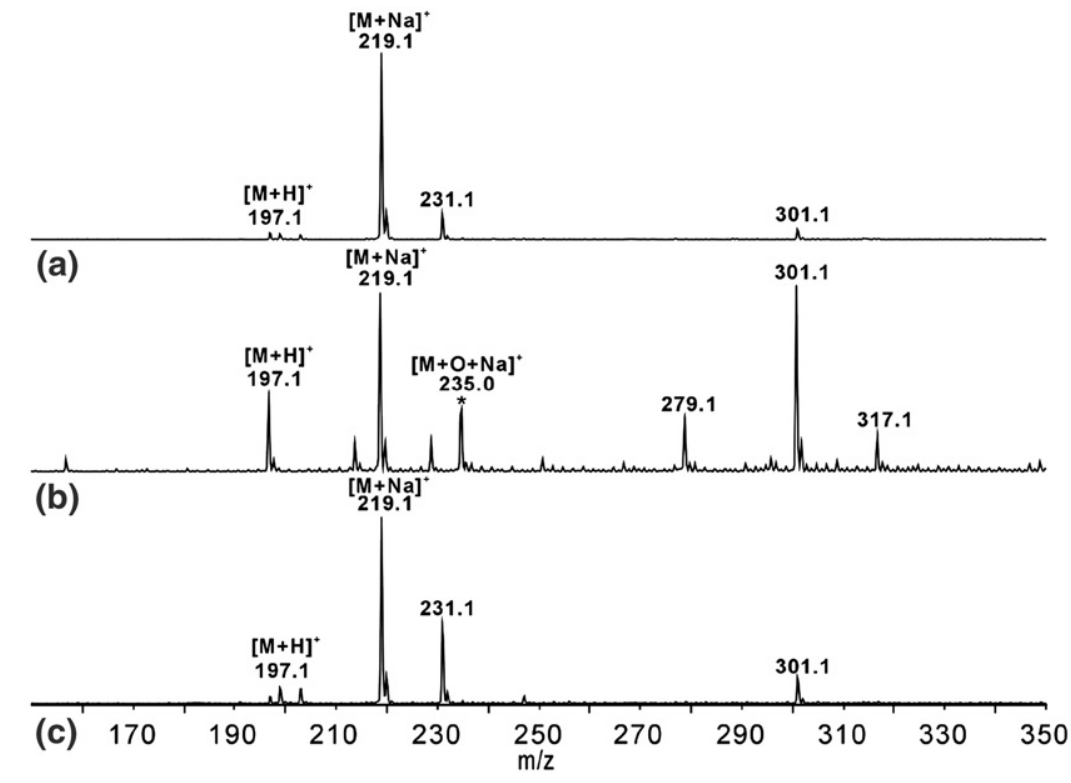

Figure 2. (a) The ESI-MS of $\mathbf{2}$ in methanol solution. (b) The DESI-MS of a newly-prepared solid pellet of $\mathbf{2}$. The star-marked signal of $\mathrm{m} / \mathrm{z} 235$ can be assigned to oxidized product of $\mathbf{2}$. (c) The ESI-MS of 2 after the DESI-monitoring.

We speculate that such a DESI-oxidation, similar to the recently reported oxidation of tamoxifen resulting from DESI process [32], may take place in the DESI ambient conditions during analyte desorption from the solid surface of the pellet containing ketone. This DESIoxidation could lead to an additional signal of the DESI-producing esters $(3,4)$ recorded in the mass spectrum overlapping on those of authentic SSORproducing esters $(3,4)$. In principle, the additional signal of the DESI-producing esters could be mathematically subtracted for better quantification through, for example, ${ }^{18} \mathrm{O}$-isotope labeled experiment. At this stage, however, it is impossible for us to conduct such an experiment because of the shortage of ${ }^{18} \mathrm{O}$ reagent $\mathrm{m}-\mathrm{ClC}_{6} \mathrm{H}_{4} \mathrm{CO}_{2}{ }^{18} \mathrm{OH}$. Note that the following profile of the SSOR kinetic curves are approximately plotted without modification.

\section{The Kinetics of Baeyer-Villiger Solid-State Organic Reactions}

The kinetic curves plotted by relative yield of the product 3 versus reaction time during the BV SSOR of 1 are shown in Figure 3a. The relative yield of 3 is defined as the molar percent of 3 divided by the sum molar percent of the reactant and product (1 and $\mathbf{3}$ ), in which the molar percent can be indirectly calculated according to the linear correlation curves between MS intensities (peak highs) and molar percents of analytes in the solid pellet (Supplementary data-6). The kinetic curve is not zero-passing, in agreement with the original oxidation of the reactant ketone to form additional DESI-producing ester product in "pure" DESI process as mentioned above. The curve displays unusual kinetics feature with variable reaction rates at three stages: (1) the velocity of
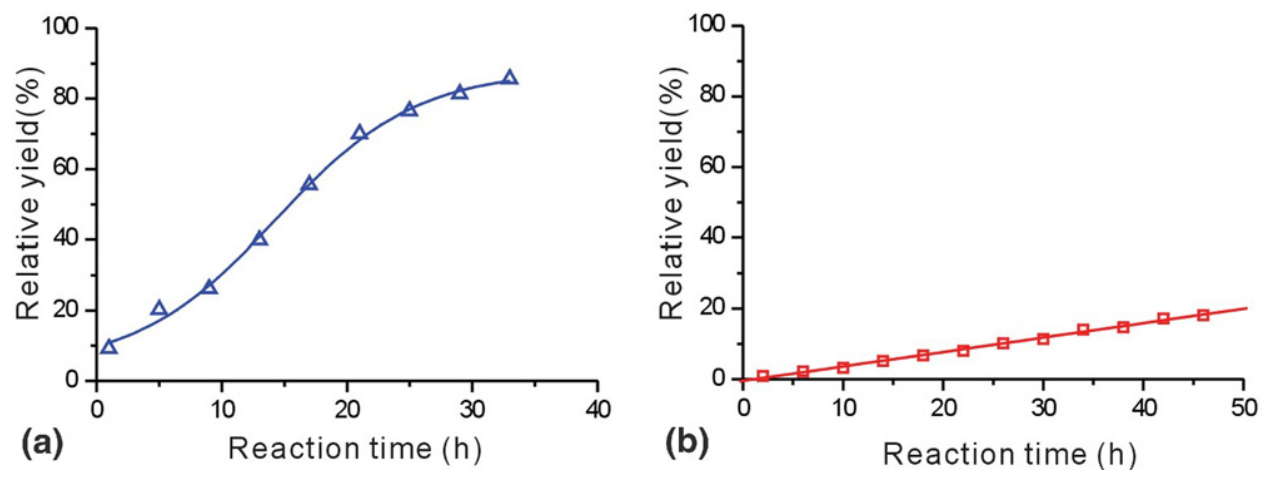

Figure 3. Kinetic curves plotted by the relative yield of product 3 as the function of BV reaction time; (a) $35 \mathrm{wt} \%$ of 1 and $65 \mathrm{wt} \%$ of m-CPBA in the solid-state; (b) $0.020 \mathrm{~g} / \mathrm{mL} \mathrm{1}$, and $0.037 \mathrm{~g} / \mathrm{mL} \mathrm{m}-\mathrm{CPBA}$ in the chloroform solution. 
the oxidation is slow in the first several hours; (2) then the oxidation becomes quicker after the 10th hour; (3) the reaction slows down at the final stage. Mathematically, this kinetic curve of the BV reaction in solid-state can be fit well with Boltzmann distribution: $y=0.87,984-0.84,263$ / $\{1+\exp [(x-14.345) / 5.6018]\}$ (Figure 3a). Similar kinetics is observed for the BV SSOR starting from 2 (Supplementary data-7).

To understand the kinetic implications of the Boltzmann equation, a control experiment with the BV reaction in solution was conducted for comparison. In agreement with previous literature [17], our experimental data also suggest the dramatically faster $\mathrm{BV}$ reaction rate in solid-state than in solution. For example, oxidations of the ketone ( 1 or 2 ) in the solid-state produce 3 in ca. $45 \%$ or 4 in ca. $85 \%$ yields after reaction for $20 \mathrm{~h}$, in contrast with the same process in chloroform solution giving products less than $10 \%$ yields. Moreover, the kinetic curves of the SSOR are different from otherwise the same reactions in chloroform solution (Figure 3 and Supplementary data-7).

The unexpected kinetic differences between the solidstate reaction and solution reaction portend that any slower operations about the reactants diffusion may influence the kinetic process significantly. In solution, the reactants are surrounded with solvent molecules. To some extent, these surrounding solvents prevent the reactants from effective collision. It is reasonable to assume the reaction being kinetically dependent on the driving force of diffusion for valid collision, which, in principle, corresponds to the concentrations of reactants and thus results in the linear kinetic curves of concentrationdependent in solution (Figure $3 \mathrm{~b}$ and Figure S10b in Supplementary data-7). By contrast, the unusual kinetics of BV SSORs may be governed by a solvent-free melting process, which is driven by co-melting of ketone reactant ( 1 or 2 ) and $\mathrm{m}-\mathrm{CPBA}$ as well as ester product (3 or 4) [33] (Supplementary data-8). In principle, the co-melting process seems to be density-insensitive, which may lead to the kinetics insensitivity even up to the $40 \mathrm{wt} \%$ loading of amylum diluents (Figure S10a in Supplementary data-7). This kind of melting process, naturally immature at the first few hours of reaction, renders the first stage of the kinetic reaction with slow velocity. With the co-melting moving on, the contacting possibility of reactant molecules increases, resulting in reactant collision easier and reaction rate faster. At the final stage of the BV SSOR, the reaction slows down because of the reduced amount of reactants, similar to the kinetic process of elementary reaction in collision theory [34]. Seemingly, the BV oxidation reactants in solid-state were observed undergoing three-stage Boltzmann-like processes.

\section{Conclusion}

In conclusion, the DESI-MS has been proven to be a useful tool for monitoring organic reaction of solid-state in ambient air. As an example, the kinetics of Baeyer-
Villiger type oxidations involving benzophenone or deoxybenzoin with m-chloroperbenzoic acid in solidstate have been quantified by DESI-MS with RSDs of around $12 \%$. It is also established that the ketone reactants can be oxidized to form ester products by the DESI process even in the absence of oxidant m-CPBA. The recorded DESI-MS data improve our understanding about the kinetics of organic reaction in solid-state, e.g., the kinetic curve, reaction rate, and concentration dependency. Similar applications may be expected using the other MS technologies recently reported to have the potential for direct analysis of solid in ambient atmosphere. The present work marks the beginning of investigation using mass spectrometry for kinetic reactions in solid-state, which may provide illumination for future studies on some important kinetic reactions involved in solid surfaces, such as heterogeneous catalysis and electrode reactions.

\section{Acknowledgments}

The authors thank Mr. Gao-Sheng Chen for his generous help in designing the DESI device. The authors acknowledge support for this work by the NSFC (no. 20525103, 20721001) and the National Basic Research Program of China (973 Program) (no. 2007CB815301).

\section{Appendix A Supplementary Material}

Supplementary material associated with this article may be found in the online version at doi:10.1016/ j.jasms.2009.07.010.

\section{References}

1. Takats, Z.; Wiseman, J. M.; Cologan, B.; Cooks, R. G. Mass Spectrometry Sampling Under Ambient Conditions with Desorption Electrospray Ionization. Science 2004, 306, 471-473.

2. Cotte-Rodriguez, I.; Takats, Z.; Talaty, N.; Chen, H.; Cooks, R. G. Desorption Electrospray Ionization of Explosives on Surfaces: Sensitivity and Selectivity Enhancement by Reactive Desorption Electrospray Ionization. Anal. Chem. 2005, 77, 6755-6764.

3. Chen, H.; Talaty, N. N.; Takats, Z.; Cooks, R. G. Desorption Electrospray Ionization Mass Spectrometry for High-Throughput Analysis of Pharmaceutical Samples in the Ambient Environment. Anal. Chem. 2005, 77 6915-6927.

4. Weston, D. J.; Bateman, R.; Wilson, I. D.; Wood, T. R.; Creaser, C. S. Direct Analysis of Pharmaceutical Drug Formulations Using Ion Mobility Spectrometry/Quadrupole-Time-of-Flight Mass Spectrometry Combined with Desorption Electrospray Ionization. Anal. Chem. 2005, 77, 7572-7580.

5. Cooks, R. G.; Ouyang, Z.; Takats, Z.; Wiseman, J. M. Ambient Mass Spectrometry. Science 2006, 311, 1566-1570.

6. Bereman, M. S.; Muddiman, D. C. Detection of Attomole Amounts of Analyte by Desorption Electrospray Ionization Mass Spectrometry (DESI-MS) Determined Using Fluorescence Spectroscopy. J. Am. Soc. Mass Spectrom. 2007, 18, 1093-1096.

7. Venter, A.; Nefliu, M.; Cooks, R. G. Ambient Desorption Ionization Mass Spectrometry. TrAC-Trends Anal. Chem. 2008, 27, 284-290.

8. Sampson, J. S.; Hawkridge, A. M.; Muddiman, D. C. Construction of a Versatile High Precision Ambient Ionization Source for Direct Analysis and Imaging. J. Am. Soc. Mass Spectrom. 2008, 19, 1527-1534.

9. Van Berkel, G. J.; Ford, M. J.; Deibel, M. A. Thin-Layer Chromatography and Mass Spectrometry Coupled Using Desorption Electrospray Ionization. Anal. Chem. 2005, 77, 1207-1215.

10. Wiseman, J. M.; Ifa, D. R.; Song, Q.; Cooks, R. G. Tissue Imaging at Atmospheric Pressure Using Desorption Electrospray Ionization (DESI) Mass Spectrometry. Angew. Chem. Int. Ed. 2006, 45, 7188-7192.

11. Ifa, D. R.; Manicke, N. E.; Dill, A. L.; Cooks, R. G. Latent Fingerprint Chemical Imaging by Mass Spectrometry. Science 2008, 321, 805. 
12. Chen, H.; Li, M.; Zhang, Y.-P.; Yang, X.; Lian, J.-J.; Chen, J.-M. Rapid Analysis of SVOC in Aerosols by Desorption Electrospray Ionization Mass Spectrometry. I. Am. Soc. Mass Spectrom. 2008, 19, 450-454.

13. Miao, Z.; Chen, H. Direct Analysis of Liquid Samples by Desorption Electrospray Ionization-Mass Spectrometry (DESI-MS). J. Am. Soc. Mass. Spectrom. 2009, 20, 10-19.

14. Cody, R. B.; Laramee, J. A.; Durst, H. D. Versatile New Ion Source for the Analysis of Materials in Open Air under Ambient Conditions. Anal. Chem. 2005, 77, 2297-2302.

15. Takats, Z.; Cotte-Rodriguez, I.; Talaty, N.; Chen, H.; Cooks, R. G. Direct, Trace Level Detection of Explosives on Ambient Surfaces by Desorption Electrospray Ionization Mass Spectrometry. Chem. Commun. 2005, 15, 1950-1952.

16. Sampson, J. S.; Hawkridge, A. M.; Muddiman, D. C. Generation and Detection of Multiply-Charged Peptides and Proteins by Matrix-Assisted Laser Desorption Electrospray Ionization (MALDESI) Fourier Transform Ion Cyclotron Resonance Mass Spectrometry. J. Am. Soc. Mass Spectrom. 2006, 17, 1712-1716.

17. Haapala, M.; Pol, J.; Saarela, V.; Arvola, V.; Kotiaho, T.; Ketola, R. A.; Franssila, S.; Kauppila, T. J.; Kostiainen, R. Desorption Atmospheric Pressure Photoionization. Anal. Chem. 2007, 79, 7867-7872.

18. Shiea, J.; Huang, M.Z. HSu, H.-J.; Lee, C.-Y.; Yuan, C.-H.; Beech, I. Sunner, J. Electrospray-Assisted Laser Desorption/Ionization Mass Spectrometry for Direct Ambient Analysis of Solids. Rapid Commun. Mass Spectrom. 2005, 19, 3701-3704.

19. McEwen, C. N.; McKay, R. G.; Larsen, B. S. Analysis of Solids, Liquids, and Biological Tissues Using Solids Probe Introduction at Atmospheric Pressure on Commercial LC/MS Instruments. Anal. Chem. 2005, 77, 7826-7831.

20. Toda, F. Solid State Organic Chemistry: Efficient Reactions, Remarkable Yields, and Stereoselectivity. Acc. Chem. Res. 1995, 28, 480-486.

21. Kaupp, G. Organic Solid-State Reactions with $100 \%$ Yield. In Organic Solid State Reactions, Toda, F., Ed.; Springer-Verlag Berlin Heidelberg: New York, 2005, p 95

22. Toda, F.; Yagi, M.; Kiyoshige, K. Baeyer-Villiger Reaction in the Solid State. J. Chem. Soc. 1988, 14, 958-959.
23. Michael Renz, B. M. 100 Years of Baeyer-Villiger Oxidations. Eur. J. Org. Chem. 1999, 1999, 737-750.

24. Rastogi, R. P.; Singh, N. B.; Singh, R. P. Organic Solid-State Reaction. J. Solid State Chem. 1977, 20, 191-200.

25. Tanaka, K.; Toda, F. Solvent-Free Organic Synthesis. Chem. Rev. 2000, 100, 1025-1074.

26. Cave, G. W. V.; Raston, C. L.; Scott, J. L. Recent Advances in Solventless Organic Reactions: Towards Benign Synthesis with Remarkable Versatility. Chem. Commun. 2001, 2159-2169.

27. Epple, M.; Sankar, G.; Thomas, J. M. Solid-State Polymerization Reaction by Combined In-Situ X-ray Diffraction and X-ray Absorption Spectroscopy (XRD-EXAFS). Chem. Mater. 1997, 9, 3127-3131.

28. Aliev, A. E.; Elizabe, L.; Kariuki, B. M.; Kirschnick, H.; Thomas, J. M.; Epple, M.; Harris, K. D. M. In Situ Monitoring of Solid-State Polymerization Reactions in Sodium Chloroacetate and Sodium Bromo acetate by ${ }^{23} \mathrm{Na}$ and ${ }^{13} \mathrm{C}$ Solid-State NMR Spectroscopy. Chem. Eur. J. 2000, 6, $1120-1126$.

29. de Miguel, Y. R.; Shearer, A. S. Infrared Spectroscopy in Solid-Phase Synthesis. Biotechnol. Bioeng. 2000, 71, 119-129.

30. Nakamatsu, S.; Yoshizawa, K.; Toyota, S.; Toda, F.; Matijasic, I. Isolation of an Inclusion Complex of Naphthol and Its Benzoate as an Intermediate in the Solvent-Free Benzoylation Reaction of Naphthol. Org. Biomol. Chem. 2003, 1, 2231-2234.

31. Ifa, D. R.; Manicke, N. E.; Rusine, A. L.; Cooks, R. G. Quantitative Analysis of Small Molecules by Desorption Electrospray Ionization Mass Spectrometry from Polytetrafluoroethylene Surfaces. Rapid Commun. Mass Spectrom. 2008, 22, 503-510.

32. Pasilis, S. P.; Kertesz, V.; Van Berkel, G. J. Unexpected Analyte Oxidation during Desorption Electrospray Ionization-Mass Spectrometry. Anal. Chem. 2008, 80, 1208-1214.

33. Rothenberg, G.; Downie, A. P.; Raston, C. L.; Scott, J. L. Understanding Solid/Solid Organic Reactions. J. Am. Chem. Soc. 2001, 123, 8701-8708.

34. Upadhyay, S. K. Chemical Kinetics and Reaction Dynamics; Springer: New York, 2006; pp. 83-88. 